\title{
Imaging of Odontogenic Infections: A Pictorial Review of What Can Happen When You Don't Floss!
}

Rebecca T. Le MD1, Mauricio Hernandez PhD¹, Thien Huynh MD1, Dinesh Rao MD¹, Jeet Patel MD ${ }^{1}{ }^{*}$

1. UF College of Medicine - Jacksonville, Department of Radiology, ${ }^{*}$ Correspondence - jeet.patel@jax.ufl.edu

\section{LEARNING OBJECTIVES}

1. Review dental anatomy (e.g. tooth and periodontal region), and dental terminology (e.g. directional terms used by dentists, American Dental Association (ADA) numbering system).

2. Review pertinent anatomic spaces affected by dental infections.

3. Illustrate a case-based approach of odontogenic infection severity, from localized dental disease to osteomyelitis.

\section{BACKGROUND}

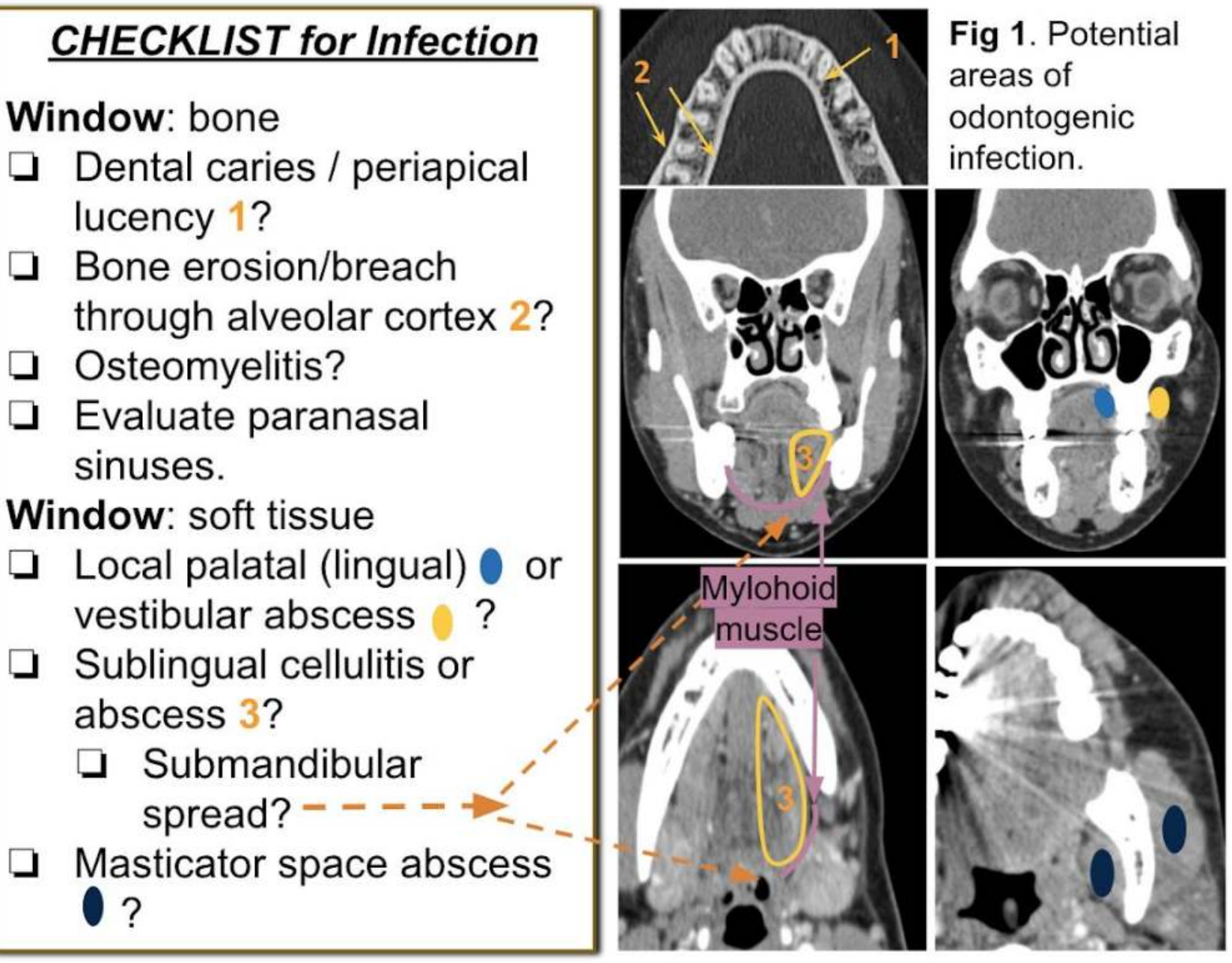

WHAT HAPPENS WHEN YOU DON'T FLOSS...
Greater than $90 \%$ of all head and neck region infections are odontogenic in origin ${ }^{1}$. Dental infections can spread to several anatomic spaces when localized disease extends past alveolar bone. Evaluation requires a comprehensive checklist to review dental structures and key complication sites, and relies on a familiarity of routes of spread.

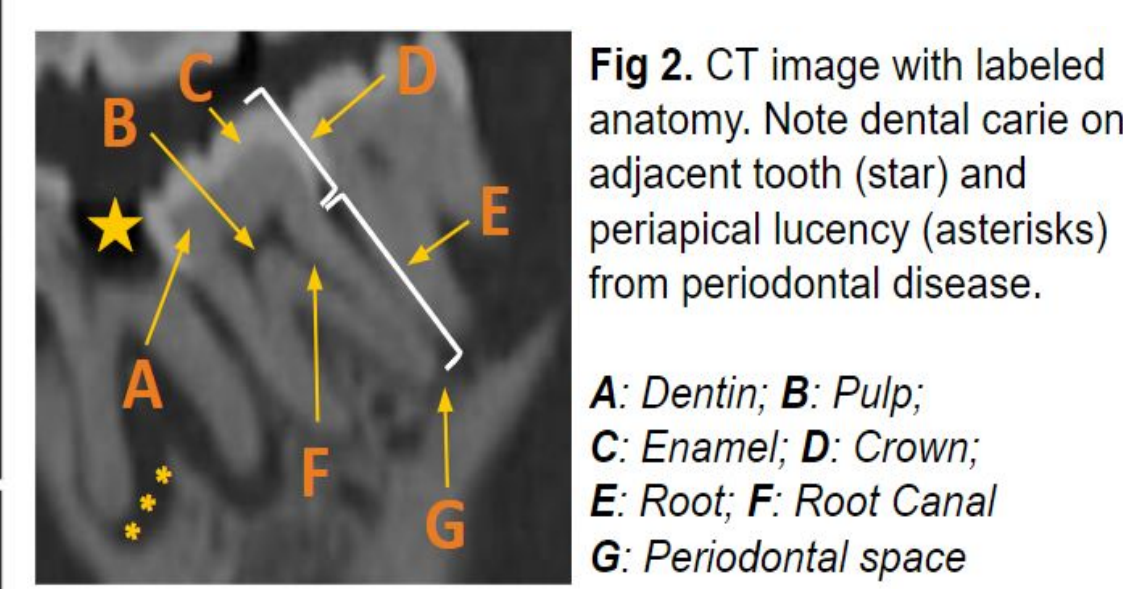

Fig 3. Dental directional/ positional terminology.

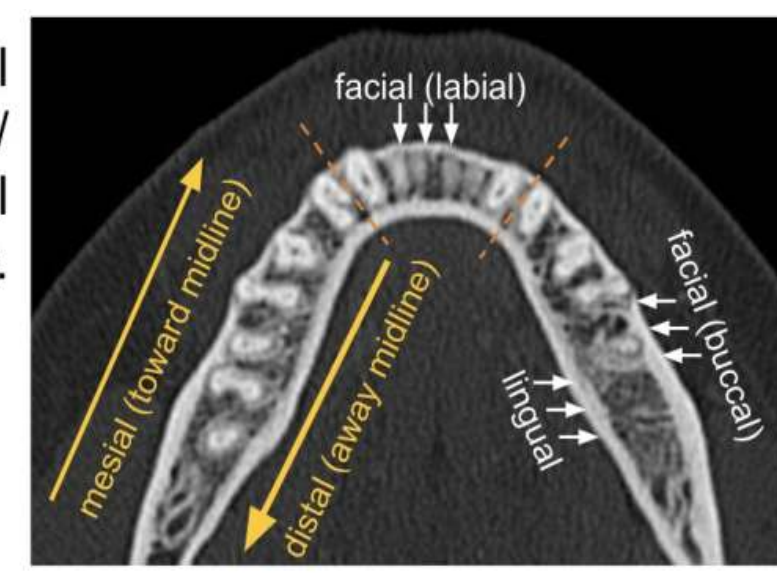

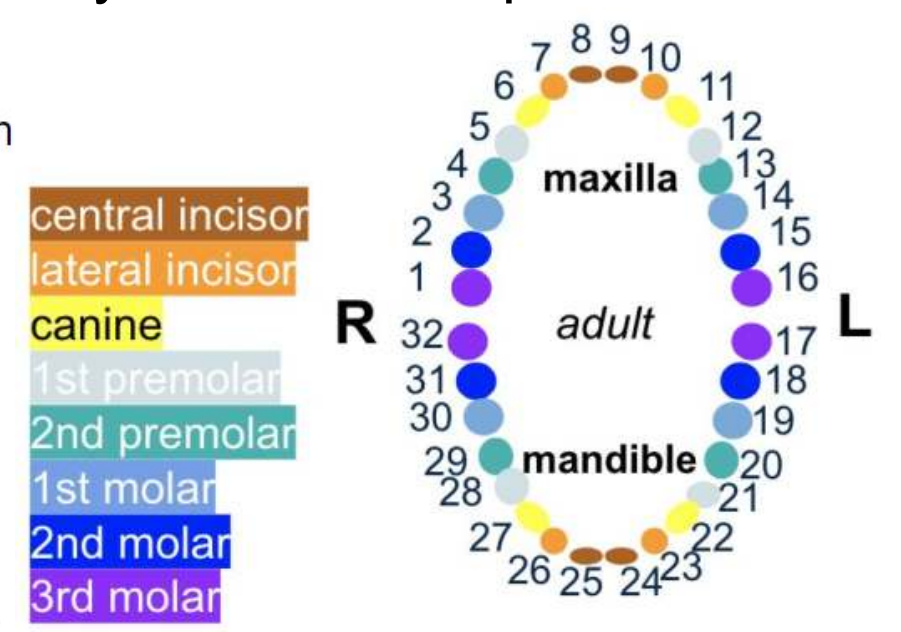

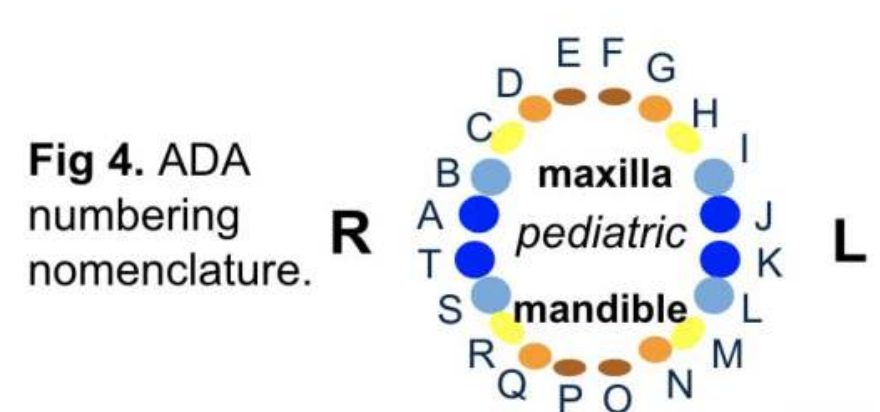

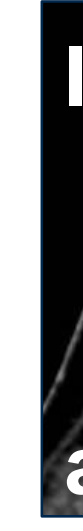

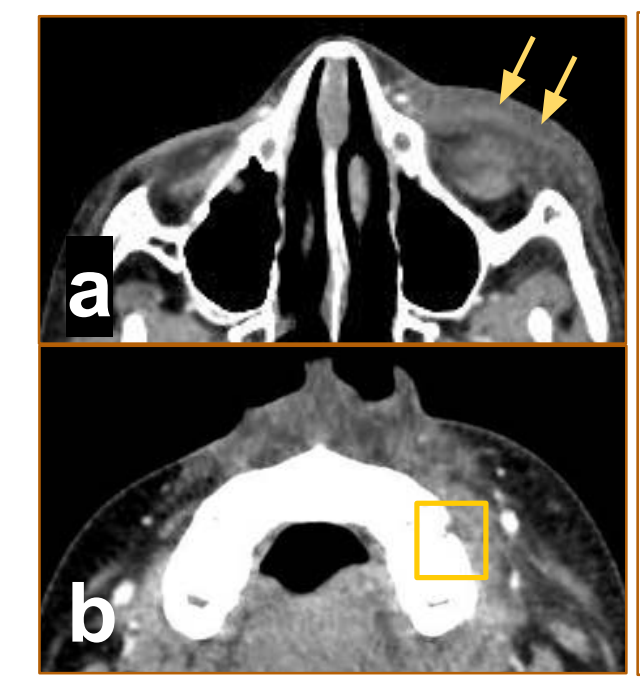

b

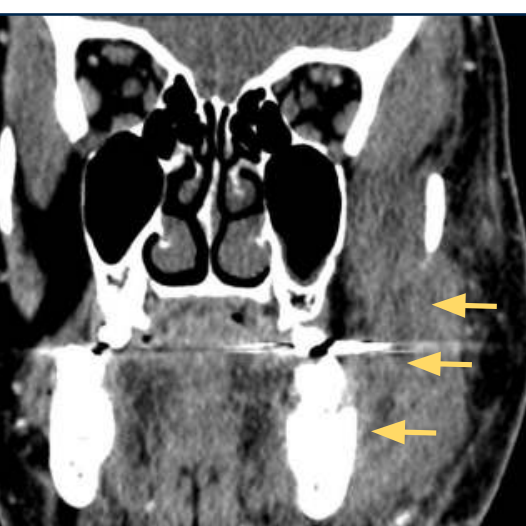

¿l. 9-year-old male presenting with periorbital cellulitis (a; arrows) associated with a draining vestibular abscess $(b$ box). He received a short course of IV antibiotics before being discharged on oral antibiotics and close follow-up with his pediatrician and dentist.

$\checkmark$ III. 49-year-old male with an odontogenic sublingual gas-forming abscess (a, b; arrow) extending posteroinferiorly around mylohyoid muscle edge into the submandibular space (c; SM = submandibular gland) causing respiratory distress and requiring same-day OR incision and drain placement. The patient continued IV antibiotics for five days and was discharged with oral antibiotics and close follow-up with oral and maxillofacial surgery (OMFS) for drain removal. Note edema and mass effect on tongue $(a, b)$ suggestive of incipient Ludwig's angina.

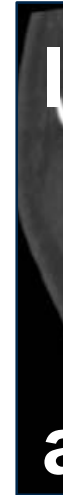
Weise $\mathrm{H}$ et al. Radiographics. 32: 1927-1944.
ه. 49 year-old male with left masticator space abscess in masseter muscle (a; arrow) extending to the zygomatic arch level (b; arrows) with associated facial cellulitis. He was admitted for IV antibiotics and discharged after 48 hours with oral antibiotics and with recommendation for close outpatient follow-up with a dentist for the causative periodontal disease.

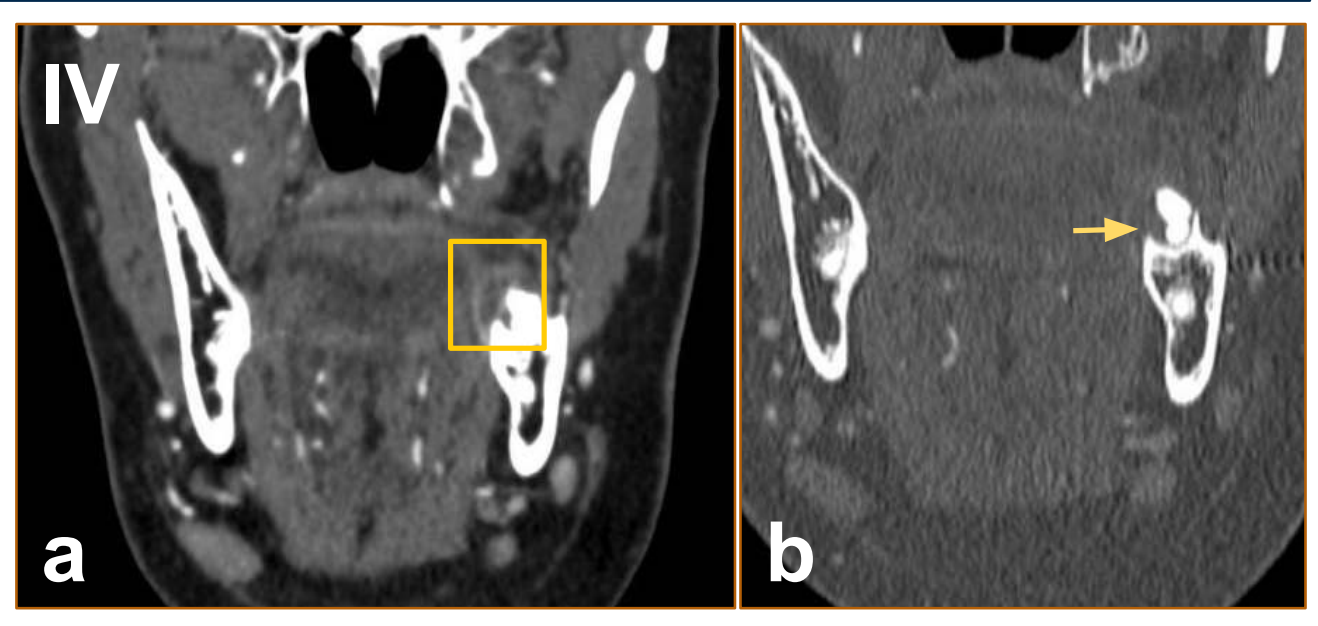

UIV. 82-year-old female with left lower jaw pain Maxillofacial CT scan was largely unremarkable, but coronal view showed a rim-enhancing odontogenic lingual gingival periosteal abscess (a; box) secondary to focal periodontal disease with periapical lucency (b; arrow). OMFS evaluated and recommended discharge with oral antibiotics and close follow-up.

Cyl. 38-year-old female with HIV (CD4=200; undetectable viral load), presenting with jaw pain. Soft tissue window (a) is unremarkable, except for mild left platysma muscle thickening (a; orange arrow) along the right mandible. Bone window illustrates erosion and periosteal reaction surrounding a decayed impacted molar (box), concerning for osteomyelitis. She was admitted for IV antibiotics and underwent tooth extraction and bone biopsy by OMFS to confirm diagnosis and was discharged on oral antibiotics.

Sv. 68-year-old male with right facial pain. CT demonstrated severe right maxillary periapical lucencies (a; arrow), right buccal odontogenic abscess (b,c; arrows), and associated adjacent right maxillary odontogenic sinusitis with complete sinus opacification (a,b; star). He received IV antibiotics and had a bedside incision and drain placement. He was discharged on oral antibiotics within 24 hours with two-day follow-up with OMFS for drain removal.

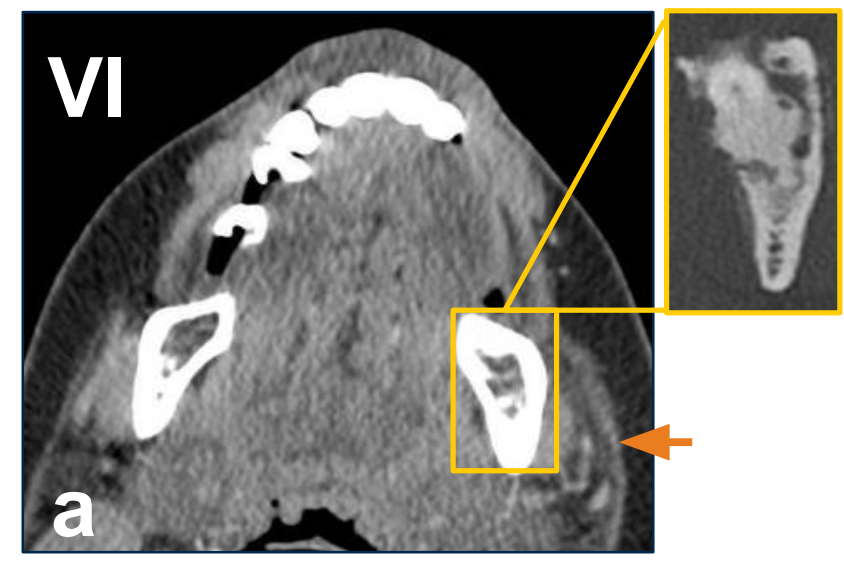

